\title{
Chemically peculiar stars as seen with 2MASS (Research Note)
}

\author{
A. Herdin ${ }^{1}$, E. Paunzen ${ }^{2}$, and M. Netopil ${ }^{2}$ \\ 1 Universitätssternwarte, Türkenschanzstr. 17, 1180 Wien, Austria \\ e-mail: epaunzen@physics .muni.cz \\ ${ }^{2}$ Department of Theoretical Physics and Astrophysics, Masaryk University, Kotlářská 2, CZ-611 37 Brno, Czech Republic
}

Received 17 September 2015 / Accepted 23 October 2015

\begin{abstract}
Context. The chemically peculiar (CP) stars of the upper main sequence are well suited for investigating the impact of magnetic fields and diffusion on the surface layers of slowly rotating stars. They can even be traced in the Magellanic Clouds and are important to the understanding of the stellar formation and evolution.

Aims. A systematic investigation of the near-infrared (NIR), 2MASS $J H K_{\mathrm{s}}$, photometry for the group of CP stars has never been performed. Nowadays, there is a great deal of data available in the NIR that reach very large distances. It is therefore very important for CP stars to be unambiguously detected in the NIR region and for these detections to be used to derive astrophysical parameters (age and mass) by applying isochrone fitting. Furthermore, we investigated whether the CP stars behave in a different way to normal-type stars in the various photometric diagrams.

Methods. For our analysis, we carefully compiled a sample of CP and apparently normal (non-peculiar) type stars. Only stars for which high-quality (i.e. with low error levels), astrometric, and photometric data are available were chosen. In total, 639 normal and $622 \mathrm{CP}$ stars were selected and further analysed. All stars were dereddened and calibrated in terms of the effective temperature and absolute magnitude (luminosity). Finally, isochrone fitting was applied.

Results. No differences in the astrophysical parameters derived from 2MASS and Johnson $U B V$ photometry were found. Furthermore, no statistical significant deviations from the normal type stars within several colour-colour and colour-magnitude diagrams were discovered. Therefore, it is not possible to detect new CP stars with the help of the photometric 2MASS colours only. A new effective temperature calibration, valid for all $\mathrm{CP}$ stars, using the $\left(V-K_{\mathrm{S}}\right)_{0}$ colour was derived.
\end{abstract}

Key words. stars: chemically peculiar - stars: early-type - techniques: photometric

\section{Introduction}

For many decades chemically peculiar (CP) stars have played an important role in stellar astrophysics. The reasons for this are mainly their average slow rotation (even among the more massive stars on the main sequence), which makes them convenient stars for measuring rotational velocities; strong magnetic fields on the upper main sequence, used to understand the role of magnetic fields in the context of stellar evolution; their photometric variability; and their peculiar chemical composition (Mikulášek et al. 2010; Krtička et al. 2013). Chemically peculiar stars were divided into four groups by Preston (1974): Am stars (CP1), Ap stars (CP2), HgMn stars (CP3), and He-weak stars (CP4). Observational progress unveiled other groups such as He-strong stars and $\lambda$ Bootis stars, and Smith (1996) created an extended classification scheme of CP stars. In addition CP2 stars can also be subdivided into several groups depending on their metal abundances (Eu, Sr, Sc, Cr, and so on). In this paper we follow the classification by Preston, but we also name the subgroups of the CP2 stars. The frequency of all CP stars in the solar neighbourhood on the main sequence ranges from 15 to $20 \%$ for stars of spectral type B5-F0 (Catalano \& Leone 1994; Romanyuk 2007).

In general, the $\mathrm{CP}$ stars seem to populate the whole age range from the zero-age main sequence (ZAMS) to the terminal-age main sequence (TAMS) which is in line with the currently used models (Pöhnl et al. 2005).

When determining fundamental parameters, photometry and spectroscopy are powerful tools to investigate these stars, though caution has to be taken when applying photometric calibrations to $\mathrm{CP}$ stars. The $u v b y \beta$ luminosity calibration (Crawford 1979) leads to deviations in absolute magnitudes of CP2 stars compared to the absolute magnitudes derived from HIPPARCOS data (Maitzen et al. 2000). This effect occurs from peculiarities in the spectra of CP2 stars, which could lead to negative $E(b-y)$ values for many stars, therefore underestimating their reddening (Figueras et al. 1998) such that additional calibration is recommended (Adelman 1979). On the other hand, Netopil et al. (2008) showed by means of cluster CP2 stars that there seems to be no significant influence on the reddening values. Unlike CP2 stars, the group of CP3 stars show no such peculiar behaviour and the $u v b y \beta$ luminosity calibration can be applied (Strömgren 1966; Figueras et al. 1998). The absolute magnitudes of CP1 stars are overestimated, hence a more accurate calibration of $M_{\mathrm{V}}$ has been developed (Figueras et al. 1998) using the extension of the ordinary least squares estimator (BCES) method as described in Akritas \& Bershady (1996) and the HIPPARCos data. Helium-weak stars have been calibrated in the BCD spectrophotometric system and the results are in good agreement with results from $U B V$ and Geneva photometry 
(Cidale et al. 2007). Helium-strong stars show different values when applying the BCD method and overestimate the effective temperature compared to the effective temperatures derived from $U B V$ and Geneva photometry (Cidale et al. 2007).

However, when it comes to infrared (IR) and near-infrared (NIR) photometry, which are less sensitive to interstellar extinction than visual observations, very little is known about CP stars. The only investigations in this respect were done by Groote \& Kaufmann (1983) and Kroll et al. (1987) who observed JHKLM fluxes for about 110 B- and A-type CP stars. Kroll et al. (1987) found no indications of dust shells or other circumstellar matter, which should manifest as an IR-excess. In addition, they found no photometric variability with an amplitude larger than 0.05 mag for their sample of stars. However, later on, variability in the NIR $(J H K)$ with the same period, spectrum, and magnetic field variations as the optical light was detected, for example, for several stars of the $\mathrm{SiSrCrEu}$ subgroup (Catalano et al. 1998). The amplitudes range from 0.04 to $0.002 \mathrm{mag}$.

In this paper, we present the first extensive study of all four CP star subgroups using the photometric data of the 2MASS survey (Skrutskie et al. 2006).

\section{Data sample}

For our analysis, we compiled a sample of CP and apparent normal-type or non-peculiar stars.

The original data sample consists of $956 \mathrm{CP}$ stars of spectral types B0 to F9 $(31 \%$ B, $66 \%$ A, $3 \%$ F) from the General Catalogue of Ap and Am stars (Renson \& Manfroid 2009). These objects are listed as best candidates in this reference and are included within the new and improved reduction of the HIPPARCos data catalogue from van Leeuwen (2007).

Our original data sample of normal stars consists of 13637 main-sequence, luminosity class $\mathrm{V}$, stars $(8 \% \mathrm{~B}, 30 \% \mathrm{~A}$, $62 \%$ F). They were chosen by the spectral classification listed by Garrison \& Gray (1994), Skiff (2014). In a series of four papers, Garrison \& Gray (1994) investigated B- to F-type stars establishing new standard stars, which is especially valuable for our paper. As a first step, we removed all objects listed as variable, peculiar, or in binary systems by cross-matching with the GCVS database (Samus et al. 2007-2014), the General Catalogue of Ap and Am stars (Renson \& Manfroid 2009), and the HIPPARCos catalogue (Perryman et al. 1997). Then, $u v b y \beta$ photometry data were taken from Hauck \& Mermilliod (1998), parallaxes from HIPPARCos data van Leeuwen (2007), NIR photometry data from 2MASS (Skrutskie et al. 2006), and visual photometry data from the All-Sky Compiled Catalogue of 2.5 million stars (ASCC, Kharchenko 2001) to produce a homogeneous data sample.

To obtain only high-quality data but still maintain a sufficiently large data sample, we restricted the error range of the data for parallaxes to $\sigma_{\pi} / \pi \leq 20 \%$ and the $1 \sigma$ errors of the broadband photometric measurements to $\sigma_{\lambda} \leq 0.1 \mathrm{mag}$ for $V J H K_{\mathrm{s}}$. The $1 \sigma$ errors of the narrowband photometric indices were constrained as follows: $(b-y) \leq 0.027 \mathrm{mag}, m_{1} \leq 0.032 \mathrm{mag}$, and $c_{1} \leq 0.050 \mathrm{mag}$ (Balona 1994). If the catalogues contained more than one measurement of a photometric index of a star, we used its mean value. Owing to insufficient $u v b y \beta$ photometric data and the lack of accuracy, most of the normal stars were removed from the original sample. In addition to this removal, further reasons for the rejection due to the inaccuracy of other data sources are (from most to least frequent) HIPPARCos parallaxes, $u v b y \beta$ dereddening, 2MASS photometry, and ASCC photometry. In addition, very few stars of the original sample were excluded for individual reasons, for example owing to an uncertain identification. Finally, 923 normal-type stars remain with high-quality data according to the above-listed standards.

Our data sample ranges in apparent magnitude and parallaxes from $4.439 \leq V \leq 10.237 \mathrm{mag}$ and $1.48 \leq \pi \leq 52.11 \mathrm{mas}$ for normal to $4.960 \leq V \leq 9.715 \mathrm{mag}$ and $2.03 \leq \pi \leq 22.70$ mas for CP stars.

\section{Analysis}

The effective temperatures for all objects were obtained using the UVBYBETA calibration by Napiwotzki et al. (1993) with the improvement for CP stars published by Netopil et al. (2008). These calibrations are well established and well tested.

We performed the dereddening for all stars also using the calibration by Napiwotzki et al. (1993). Objects for which the dereddening did not converge after 500 iterations were excluded from further analysis. These objects are mostly of luminosity class I, II, and III. To obtain the interstellar extinction-free apparent magnitudes in the NIR, we used a standard value for $E(B-V)=1.35 E(b-y)($ Crawford 1975). Stars with $E(b-y)<0$ (33.3\% of the sample, i.e. 165 normal and $237 \mathrm{CP}$ stars) were treated as exhibiting no extinction, and $E(b-y)$ was set to zero. The final data sample was reduced to 639 normal stars of spectral type B1.5 to F9 (28\% B, 23\% A, and $49 \% \mathrm{~F})$ and $622 \mathrm{CP}$ stars of spectral type B2 to F9 $(32 \% \mathrm{~B}, 64 \% \mathrm{~A}$, and $3 \% \mathrm{~F})$. The latter group consists of $263 \mathrm{CP} 1,258 \mathrm{CP} 2,63 \mathrm{CP} 3$, and $38 \mathrm{CP} 4$ stars.

The astrometric parallaxes of the HIPPARCos catalogue (van Leeuwen 2007) were transformed to absolute magnitudes $\left(M_{K_{\mathrm{S}}}\right)$ using the visual magnitude $\left(K_{\mathrm{S}}\right)$ from Skrutskie et al. (2006) and the reddening $\left(A_{K_{\mathrm{S}}}=0.50 E(b-y)\right)$ according to $M_{K_{\mathrm{S}}}=5 \log \pi+K_{\mathrm{S}}-A_{K_{\mathrm{S}}}+5$. The luminosities were calculated using the bolometric corrections from Netopil et al. (2008) scaled to $M_{\mathrm{K}_{\mathrm{S}}}$ (Masana et al. 2006).

Figure 1 shows the $M_{K_{\mathrm{S}}}$ versus $\left(V-K_{\mathrm{S}}\right)_{0}$ diagrams for all $\mathrm{CP}$ subgroups together with the isochrones from Girardi et al. (2002). The latter range from $7.0<\log t<9.5$ dex, i.e. the full range of the main-sequence for B- to F-type stars. We chose this set of isochrones because it is well tested for a wide variety of different star groups. However, we also used one set of isochrones from the newer PARSEC ${ }^{1}$ database. As expected, the differences are negligible. From the $622 \mathrm{CP}$ stars only about $3 \%$ are below the ZAMS. This lends confidence not only in the use of the 2MASS colours, but also in the applied calibrations. In Table 1 , the ZAMS for solar metallicity $(Z=0.019)$ in terms of the mass $M_{K_{\mathrm{S}}}$ and $\left(V-K_{\mathrm{S}}\right)_{0}$ is listed. Our sample roughly covers the mass range from 1.3 to $9.0 M_{\odot}$. In the following, we discuss Fig. 1 in more detail.

- CP1: almost all objects are older than $\log t>8.5$ dex with only a few objects close to the ZAMS. This is consistent with the diffusion model that explains the observed chemical peculiarities. It is more efficient in later stellar evolutionary stages and when it has a longer time to work (Michaud et al. 1983).

- CP2: the members of this group populate the whole age range from the ZAMS to about $\log t=9.3$ dex. This is in line with the conclusions drawn by Pöhnl et al. (2005) who found that at least $16 \%$ of the investigated $\mathrm{CP} 2$ stars have fractional ages below $20 \%$.

1 http://stev.oapd.inaf.it/cgi-bin/cmd 


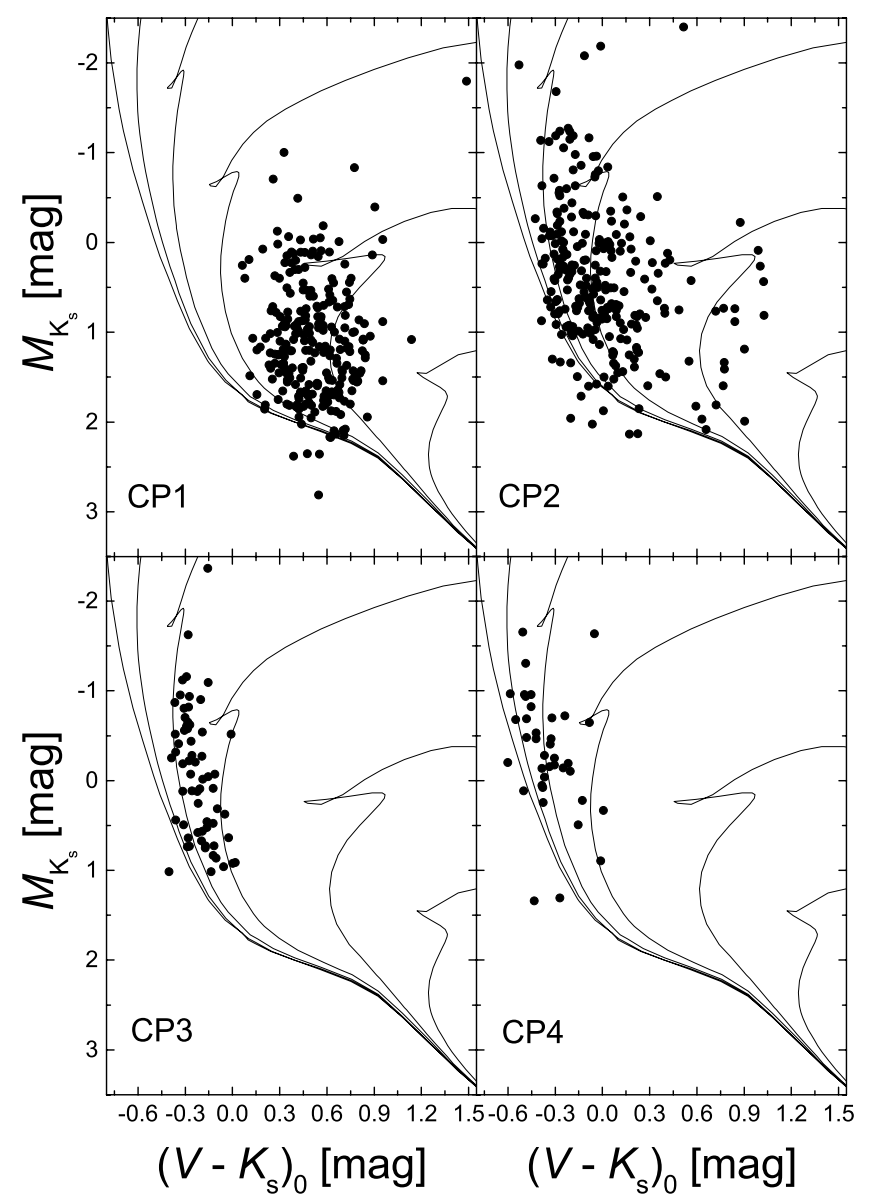

Fig. 1. $M_{K_{\mathrm{S}}}$ versus $\left(V-K_{\mathrm{S}}\right)_{0}$ diagrams for all $\mathrm{CP}$ subgroups together with the isochrones from Girardi et al. (2002).

- CP3: this is the most homogeneous group in term of the age. Almost all the stars are located in the range $8.0<$ $\log t<8.5$ dex. There are only a few stars at or close to the ZAMS.

- CP4: there is wide range of ages from the ZAMS to about 8.5 dex, which covers the complete stellar evolutionary stages for the given mass range.

The stellar fractional main-sequence ages $\left(t_{\text {frac }}\right)$ were derived using the effective temperature and luminosity by interpolating in the evolutionary tracks by Schaller et al. (1992) for solar metallicity $(Z=0.020)$. This technique was already successfully applied to all CP subgroups (Wraight et al. 2012; Paunzen et al. 2013). For the 2MASS system, the isochrones were taken from Girardi et al. (2002) for solar metallicity $(Z=0.019)$ because the ones by Schaller et al. (1992) are not available for the NIR region. Applying the above-mentioned method to the grids by Girardi et al. (2002) yielded compatible results for a representative sample of all subgroups within the errors of the effective temperatures and luminosities. For stars located outside the grids (below or above the main sequence), we adopted values for $t_{\text {frac }}$ of either zero or $100 \%$. However, more than $90 \%$ of all CP stars are located well within the used evolutionary main-sequence borders. This supports the current knowledge that $\mathrm{CP}$ stars are in general main-sequence, luminosity class $\mathrm{V}$, objects. We used the $\left(V J H K_{\mathrm{s}}\right)_{0}$ colours to get effective temperatures, luminosities, and fractional ages by simply using the smallest distance in four-dimensional space within the solar abundant isochrones.
Table 1. ZAMS (Girardi et al. 2002) for solar metallicity $(Z=0.019)$ in terms of $M_{K_{\mathrm{S}}}$ and $\left(V-K_{\mathrm{S}}\right)_{0}$ for the investigated mass range of B- to F-type stars.

\begin{tabular}{cccccc}
\hline \hline$M_{\odot}$ & $\begin{array}{c}M_{K_{\mathrm{S}}} \\
{[\mathrm{mag}]}\end{array}$ & $\begin{array}{c}\left(V-K_{\mathrm{S}}\right)_{0} \\
{[\mathrm{mag}]}\end{array}$ & $M_{\odot}$ & $\begin{array}{c}M_{K_{\mathrm{S}}} \\
{[\mathrm{mag}]}\end{array}$ & $\begin{array}{c}\left(V-K_{\mathrm{S}}\right)_{0} \\
{[\mathrm{mag}]}\end{array}$ \\
\hline 1.3 & +2.64 & +1.081 & 4.0 & +0.38 & -0.409 \\
1.4 & +2.40 & +0.923 & 4.5 & +0.16 & -0.460 \\
1.5 & +2.24 & +0.752 & 5.0 & -0.06 & -0.502 \\
1.6 & +2.11 & +0.559 & 5.5 & -0.27 & -0.539 \\
1.7 & +1.99 & +0.385 & 6.0 & -0.46 & -0.573 \\
1.8 & +1.90 & +0.244 & 6.5 & -0.64 & -0.604 \\
1.9 & +1.81 & +0.142 & 7.0 & -0.81 & -0.632 \\
2.0 & +1.70 & +0.062 & 7.5 & -0.98 & -0.657 \\
2.1 & +1.60 & -0.002 & 8.0 & -1.14 & -0.680 \\
2.2 & +1.51 & -0.054 & 8.5 & -1.32 & -0.701 \\
2.3 & +1.44 & -0.095 & 9.0 & -1.48 & -0.720 \\
2.4 & +1.38 & -0.128 & 9.5 & -1.64 & -0.736 \\
2.5 & +1.32 & -0.157 & 10.0 & -1.78 & -0.750 \\
2.6 & +1.25 & -0.184 & 10.5 & -1.92 & -0.763 \\
2.7 & +1.18 & -0.209 & 11.0 & -2.07 & -0.774 \\
2.8 & +1.11 & -0.232 & 11.5 & -2.23 & -0.783 \\
2.9 & +1.03 & -0.253 & 12.0 & -2.39 & -0.792 \\
3.0 & +0.96 & -0.273 & 12.5 & -2.54 & -0.799 \\
3.5 & +0.65 & -0.350 & 13.0 & -2.69 & -0.805 \\
\hline & & & & & \\
\hline
\end{tabular}

Although this is not a very accurate method, it is a good test if the 2MASS colours are useable for the calibration of CP stars. The comparison with the values from the classical technique yielded the following median values of the differences for the complete CP star sample: $\Delta T_{\text {eff }}=+90 \mathrm{~K}, \Delta \log L / L_{\odot}=+0.01 \mathrm{dex}$, and $\Delta t_{\text {frac }}=-5 \%$. Splitting up the sample into the four subgroups did not result in any further improvements. From a statistical point of view, the 2MASS colours can be used for CP stars to derive astrophysical parameters. This is especially important for studying more distant and therefore fainter members of this group.

The distributions of the effective temperatures, luminosities, and calibrations of the effective temperature and luminosity of the CP and normal-type objects were compared. We performed a Student's t-test (Edgington \& Onghena 2007) if both samples differed significantly from each other. On a $99.9 \%$ significance level, the distributions of the two samples do not differ. Again, this supports the basic assumption that the $\mathrm{CP}$ mechanism works from the ZAMS to the TAMS.

We inspected all colour-magnitude diagrams in the 2MASS system in order to search for a clear separation of CP to normal stars. Such a discrimination is observed in the Johnson $U B V$ and Geneva seven-colour photometric systems (Hauck \& North 1982) mainly because most CP stars exhibit a UV flux deficiency and have several flux depressions such as the most prominent at $5200 \AA$ (Kodaira 1969; Leckrone 1973). In addition, the reddening free $Q\left(J H K_{\mathrm{S}}\right)$ parameter (Comerón \& Pasquali 2012), which is defined as $Q\left(J H K_{\mathrm{S}}\right)=(J-H)+\alpha\left(H-K_{\mathrm{S}}\right)$ with $\alpha=\frac{E(J-H)}{E\left(H-K_{\mathrm{S}}\right)}$, was investigated. The actual values of $\alpha$ range between 1.4 and 2.0 (Yuan et al. 2013). No statistical significant deviation from the normal-type stars was discovered. Therefore, it is not possible to detect new CP stars with the help 

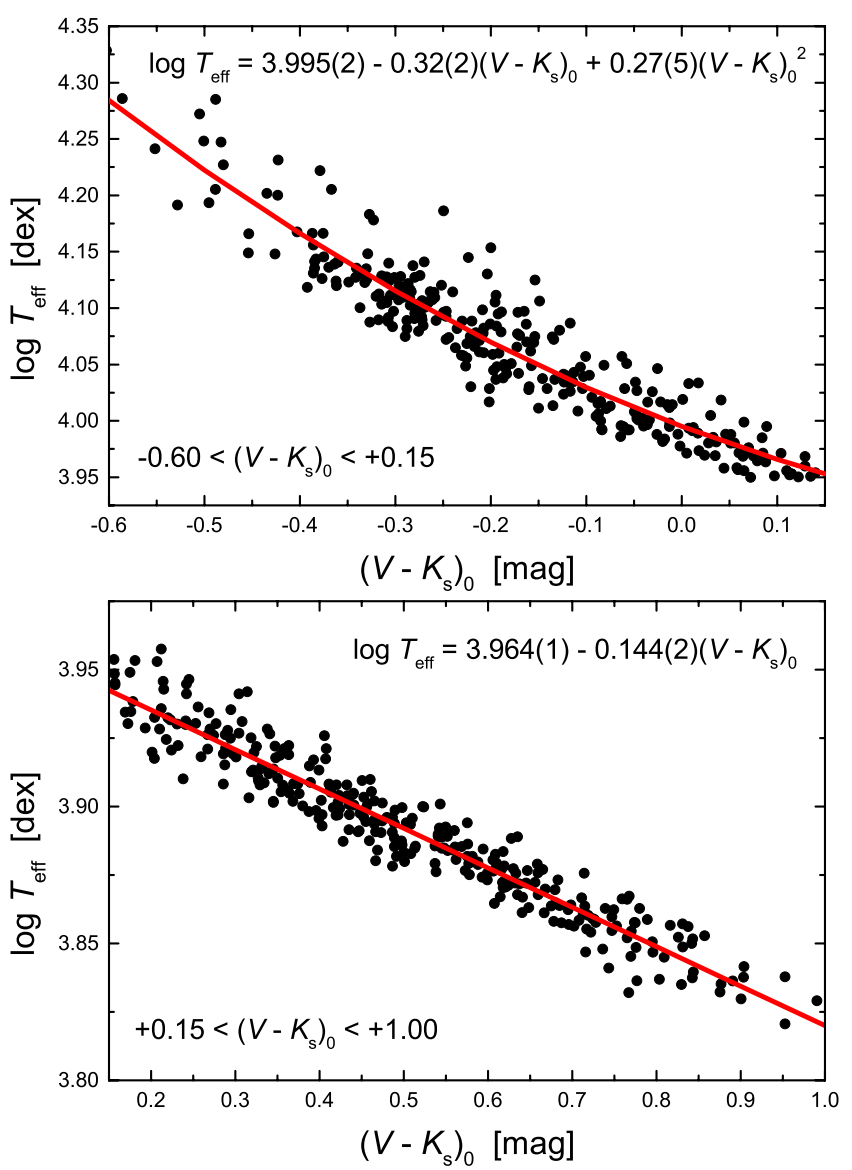

Fig. 2. Effective temperature calibration for all four $\mathrm{CP}$ star groups in terms of $\left(V-K_{\mathrm{S}}\right)_{0}$.

of the 2MASS colours only. Leone \& Catalano (1991) compared several $U B V J H$ colour-colour diagrams of normal and CP2 stars and came to a similar conclusion.

As the final step, the effective temperatures derived from the $u v b y \beta$ photometric data were plotted versus the $\left(V-K_{\mathrm{S}}\right)_{0}$ colours in order to derive a temperature calibration for CP stars. There are only a few stars that significantly deviate from the relation shown in Fig. 2. These objects are, with a few exceptions, close or spectroscopic binary systems of the CP1 subgroup and were discarded from the analysis. We fitted a line for the cooler stars and a polynomial of first order to the hotter stars for the complete sample not divided into subgroups (Fig. 2). The derived relations and their valid colour ranges are

$$
\begin{aligned}
- & \log T_{\mathrm{eff}}=3.995(2)-0.32(2)\left(V-K_{\mathrm{S}}\right)_{0}+0.27(5)\left(V-K_{\mathrm{S}}\right)_{0}^{2} \\
& \text { for }-0.60<\left(V-K_{\mathrm{S}}\right)_{0}<+0.15 \mathrm{mag} ; N=294 \\
- & \log T_{\mathrm{eff}}=3.964(1)-0.144(2)\left(V-K_{\mathrm{S}}\right)_{0} \text { for }+0.15<(V- \\
& \left.K_{\mathrm{S}}\right)_{0}<+1.00 \mathrm{mag} ; N=301 .
\end{aligned}
$$

These calibrations cover an effective temperature range from 6600 to $19000 \mathrm{~K}$. Taking the limiting colour magnitudes for both calibrations, we find a maximum error of $\pm 13 \%$ and $\pm 3 \%$, respectively. Merging both subsamples and performing a polynomial fit up to the third order did not result in a smaller error.

\section{Conclusions}

Current estimates suggest that about $15 \%$ of all B- to F-type stars belong to $\mathrm{CP}$ groups. To date, more than 8000 probable
CP objects (or candidates) are known in the Milky Way (Renson \& Manfroid 2009), of which nearly one-half belong to CP1 (i.e. non-magnetic) and CP2/4 (i.e. magnetic) stars. The group of CP3 (HgMn) objects play a minor part with only about 200 candidates listed in the aforementioned reference.

The group of CP stars is an excellent laboratory with which to test astrophysical mechanisms such as diffusion (both microscopic and turbulent), convection, meridional circulation, mass loss, and accretion in the absence/presence of an organized stellar magnetic field.

For the first time we investigated statistically the behaviour of the CP stars in the NIR using the 2MASS $J H K_{\mathrm{S}}$ colours together with the Johnson $V$ data. A sample of CP and normaltype stars was selected on the basis of available Strömgren $u v b y \beta$ colours and accurate HIPPARCOS parallaxes.

All stars were dereddened and calibrated in terms of the effective temperature and absolute magnitude (luminosity) using well-tested calibrations. A new effective temperature calibration for CP stars using the $\left(V-K_{\mathrm{S}}\right)_{0}$ colour was derived.

We conclude that the 2MASS colours are well suited to statistically deriving the astrophysical parameters of CP stars, but do not allow to distinguish them a priori from normal-type objects. However, one has to keep in mind that several subgroups of these stars are variable over the whole spectral range, which introduces an additional error source for the calibration process. This result is important for the study of more distant and therefore faint $\mathrm{CP}$ candidates for which no photometric data other than those from Johnson $V$ and 2MASS are available.

Acknowledgements. This project is financed by the SoMoPro II programme (3SGA5916). The research leading to these results has acquired a financial grant from the People Programme (Marie Curie action) of the Seventh Framework Programme of EU according to the REA Grant Agreement No. 291782. The research is further co-financed by the South-Moravian Region. It was also supported by the grants GP14-26115P, 7AMB14AT015, and the financial contributions of the Austrian Agency for International Cooperation in Education and Research (BG-03/2013 and CZ-09/2014). This work reflects only the author's views and the European Union is not liable for any use that may be made of the information contained therein.

\section{References}

Adelman, S. J. 1979, AJ, 84, 857

Akritas, M. G., \& Bershady, M. A. 1996, ApJ, 470, 706

Balona, L. A. 1994, MNRAS, 268, 119

Catalano, F. A., \& Leone, F. 1994, in The MK Process at 50 Years: a powerful tool for astrophysical insight, eds. C. J. Corbally, R. O. Gray, \& R. F. Garrison, ASP Conf. Ser., 60, 110

Catalano, F. A., Leone, F., \& Kroll, R. 1998, A\&AS, 131, 63

Cidale, L. S., Arias, M. L., Torres, A. F., et al. 2007, A\&A, 468, 263

Comerón, F., \& Pasquali, A. 2012, A\&A, 543, A101

Crawford, D. L. 1975, AJ, 80, 955

Crawford, D. L. 1979, AJ, 84, 1858

Edgington, E. S., \& Onghena, P. 2007, Randomization Tests, Fourth Edition (London: Chapman and Hall/CRC)

Figueras, F., Luri, X., Gomez, A. E., et al. 1998, Contributions of the Astronomical Observatory Skalnate Pleso, 27, 184

Garrison, R. F., \& Gray, R. O. 1994, AJ, 107, 1556

Girardi, L., Bertelli, G., Bressan, A., et al. 2002, A\&A, 391, 195

Groote, D., \& Kaufmann, J. P. 1983, A\&AS, 53, 91

Hauck, B., \& Mermilliod, M. 1998, A\&AS, 129, 431

Hauck, B., \& North, P. 1982, A\&A, 114, 23

Kharchenko, N. V. 2001, Kinematika i Fizika Nebesnykh Tel, 17, 409

Kodaira, K. 1969, ApJ, 157, 59

Kroll, R., Schneider, H., Voigt, H. H., \& Catalano, F. A. 1987, A\&AS, 67, 195

Krtička, J., Janík, J., Marková, H. et al. 2013, A\&A, 556, A18

Leckrone, D. S. 1973, ApJ, 185, 577

Leone, F., \& Catalano, F. A. 1991, A\&A, 242, 199 
A. Herdin et al.: Chemically peculiar (CP) stars as seen with 2 MASS $(R N)$

Maitzen, H. M., Paunzen, E., Vogt, N., \& Weiss, W. W. 2000, A\&AS, 355, 1003 Masana, E., Jordi, C., \& Ribas, I. 2006, A\&A, 450, 735

Michaud, G., Tarasick, D., Charland, Y., \& Pelletier, C. 1983, ApJ, 269, 239

Mikulášek, Z., Krtička, J., Henry, G. W., et al. 2010, A\&A, 511, L7

Napiwotzki, R., Schoenberner, D., \& Wenske, V. 1993, A\&A, 268, 653

Netopil, M., Paunzen, E., Maitzen, H. M., North, P., \& Hubrig, S. 2008, A\&A, 491, 545

Paunzen, E., Wraight, K. T., Fossati, L., et al. 2013, MNRAS, 429, 119

Perryman, M. A. C., Lindegren, L., Kovalevsky, J., et al. 1997, A\&A, 323, L49

Pöhnl, H., Paunzen, E., \& Maitzen, H. M. 2005, A\&A, 441, 1111

Preston, G. W. 1974, ARA\&A, 12, 257

Renson, P., \& Manfroid, J. 2009, A\&A, 498, 961

Romanyuk, I. I. 2007, Astrophys. Bull., 62, 62
Samus, N. N., Durlevich, O. V., Kazarovets, E. V., et al. 2007-2014, General Catalogue of Variable Stars, VizieR On-line Catalog, http://cdsarc. u-strasbg.fr/viz-bin/Cat?B/gcvs

Schaller, G., Schaerer, D., Meynet, G., \& Maeder, A. 1992, A\&AS, 96, 269

Skiff, B. A. 2014, Catalogue of Stellar Spectral Classifications, VizieR Online Data Catalog, http://cdsarc.u-strasbg.fr/viz-bin/VizieR? - source $=B / \mathrm{mk}$

Skrutskie, M. F., Cutri, R. M., Stiening, R., et al. 2006, AJ, 131, 1163

Smith, K. C. 1996, Ap\&SS, 237, 77

Strömgren, B. 1966, ARA\&A, 4, 433

van Leeuwen, F. 2007, A\&A, 474, 653

Wraight, K. T., Fossati, L., Netopil, M., et al. 2012, MNRAS, 420, 757

Yuan, H. B., Liu, X. W., \& Xiang, M. S. 2013, MNRAS, 430, 2188 